\title{
Optimized Diffusion of Run-and-Tumble Particles in Crowded Environments
}

\author{
Thibault Bertrand, ${ }^{1, *}$ Yongfeng Zhao, ${ }^{2}$ Olivier Bénichou, ${ }^{3}$ Julien Tailleur, ${ }^{2}$ and Raphaël Voituriez ${ }^{1,3, \dagger}$ \\ ${ }^{1}$ Laboratoire Jean Perrin, UMR 8237 CNRS, Sorbonne Université, 75005 Paris, France \\ ${ }^{2}$ Laboratoire Matière et Systèmes Complexes, UMR 7057 CNRS, Université Paris Diderot, 75205 Paris, France \\ ${ }^{3}$ Laboratoire de Physique Théorique de la Matière Condensée, \\ UMR 7600 CNRS, Sorbonne Université, 75005 Paris, France
}

(Dated: July 12, 2018)

\begin{abstract}
We study the transport of self-propelled particles in dynamic complex environments. To obtain exact results, we introduce a model of run-and-tumble particles (RTPs) moving in discrete time on a $d$-dimensional cubic lattice in the presence of diffusing hard-core obstacles. We derive an explicit expression for the diffusivity of the RTP, which is exact in the limit of low density of fixed obstacles. To do so, we introduce a generalization of Kac's theorem on the mean return times of Markov processes, which we expect to be relevant for a large class of lattice gas problems. Our results show the diffusivity of RTPs to be nonmonotonic in the tumbling probability for low enough obstacle mobility. These results prove the potential for optimization of the transport of RTPs in crowded and disordered environments with applications to motile artificial and biological systems.
\end{abstract}

Run-and-tumble particles (RTPs) are a prototypical model of self-propelled particles (SPPs) at the colloidal scale, which belongs to the broader class of active matter systems [1,2]. In its simplest form, RTP trajectories consist of a sequence of randomly oriented "runs"- periods of persistent motion in a straight line at a constant speed-interrupted by instantaneous changes of direction, called "tumbles", occurring at random with a constant rate. This canonical model has played a pivotal role in the theoretical description of self-propelled biological entities such as bacteria [3-7], algae [8], eukaryotic cells [9], or larger scale animals [10].

Whereas systems in thermal equilibrium display a timereversal symmetry, this invariance is generically lost in active matter at the microscopic scale because of the continuous consumption of energy. Nevertheless, at a constant speed and tumbling rate, an isolated RTP performs a random walk with diffusive scaling at large enough time- and length scales which cannot be qualitatively distinguished from the equilibrium dynamics of Brownian colloids. Hence, it is only through their interactions with either other particles or the environment that RTPs display non-equilibrium features. Interactions between SPPs can indeed have spectacular consequences which have attracted a growing interest over the last decade. For instance, dense active suspensions can display large-scale collective motion in settings where long-range order would be forbidden for equilibrium systems [11-14]. Another non-thermal collective effect is the propensity of active particles to cluster [15-17] or undergo phase-separation [18-20] in the presence of purely repulsive interactions.

The interplay between active particles and their environment has also attracted a lot of interest [2]. Most motile biological systems such as bacteria or dendritic cells navigate disordered and complex natural environments such as soils, soft gels (e.g. mucus or agar) or tissues. Recent simulations have explored the dynamics of active particles in the presence of quenched disorder as well as active baths [21-25]. In confined geometries, active particles accumulate at the boundaries, at odds with the equilibrium Boltzmann distribution. This has been observed for a variety of systems from spher- ical and elongated particles in linear channels to bacteria in spherical cavities [26-31]. Such non trivial interactions with obstacles can lead to effective trapping and thus have important consequences in the dynamics of SPPs in disordered environments. Indeed, active particles in the presence of static obstacles can display subdiffusive dynamics [32]. Trapping has been observed both in models [33-35] and in experiments of biological or synthetic microswimmers [36-38]. It was shown in numerical simulations of RTPs moving through arrays of obstacles that trapping can lead to the existence of an optimal activity level for drift through the system [39, 40]. More recently, the presence of disordered obstacles was shown to destroy the emergence of large scale correlations preventing flocking and swarming [41]. Despite these various observations, the generic analysis of the dynamics of a single SPP in disordered environments remains mostly unexplored, and in particular analytical results are largely missing.

In this letter, we introduce a minimal model of discrete time RTP moving on a $d$-dimensional cubic lattice in the presence of diffusing hard-core obstacles of density $\rho$, which model a potentially dynamic disordered environment. In particular, this generalizes to RTPs questions that have attracted a lot of attention for passively diffusing particles [42] and externally driven tracers [43-46]. We determine analytically numerous observables characterising the dynamics : the mean free run time, defined as the mean time between consecutive collisions of the RTP with obstacles, the mean trapping time of the RTP by obstacles, and the large-scale diffusion coefficient of the RTP. This calculation is exact for fixed obstacles in the limit of low obstacle density $\rho \rightarrow 0$, and remains uniformly accurate in the tumble rate for finite values of $\rho$ and mobile obstacles. Our analysis reveals the existence of a maximum of the diffusion coefficient of the RTP as a function of the tumbling rate, for a low enough mobility of obstacles. Our approach is based on a generalization of a theorem due to Kac on mean return times of Markov processes [47], which was already shown to have important applications in physics [48, 49]. We show here that it implies the following exact result: For fixed obstacles the mean free run time is given by $\left\langle\tau_{r}\right\rangle=1 / \rho$, and is indepen- 
dent of the tumbling rate of the RTP. In addition, in the case of moving obstacles, this result still holds for a proper choice of microscopic collision rules and is independent of the diffusion coefficient of the obstacles, provided that the time step of the obstacle dynamics is larger than that of the RTP. This strikingly simple result has the potential to find a variety of applications in general lattice gas problems.

Model and definitions - We consider a discrete time RTP on an infinite lattice in $d$ dimensions surrounded by obstacles uniformly distributed with a density $\rho$ as shown in Fig. 1 . The RTP, of position $\boldsymbol{r}(t)$, is polarized in a given direction and, in absence of interaction with obstacles, makes one lattice step per unit time in this direction (run) until its polarity is reset randomly amongst the $2 d$ possible directions on the lattice (tumble). We consider that these tumbling events happen at each time step with probability $\alpha$, independently of the presence of obstacles. We assume that the obstacles perform

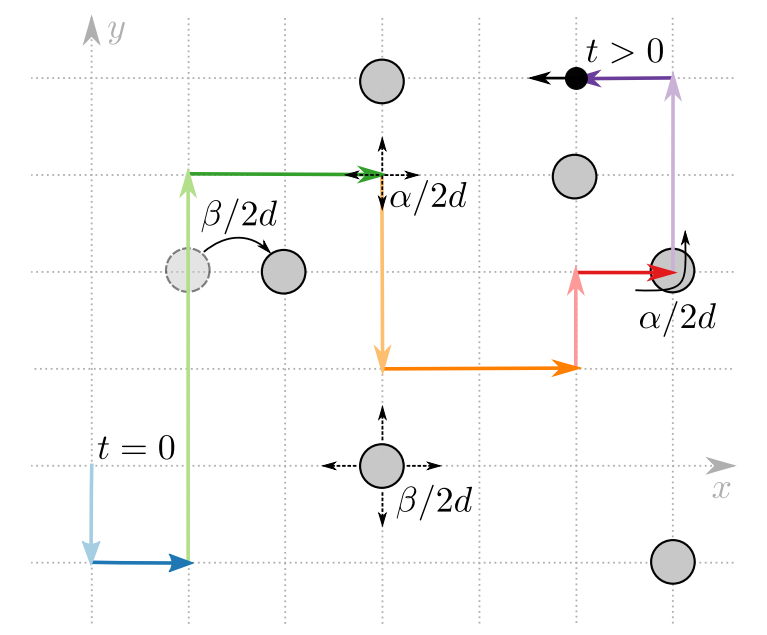

FIG. 1. Example trajectory of a run-and-tumble particle (black) on a 2D lattice among uniformly distributed obstacles (gray) at density $\rho$. At $t=0$, the RTP starts from the origin and it moves along the direction of its polarity (black arrow) in a sequence of linear runs (pictured here in different colors) punctuated by encounters with obstacles and tumbles. The obstacles have a probability $\beta / 2 d$ to move in a given direction and the RTP has a probability $\alpha / 2 d$ to flip its polarity along a particular direction, potentially untrapping the RTP.

symmetric nearest neighbour random walks, with probability $\beta<1$ to jump at each time step. We consider obstacles interacting via hard-core repulsion; i.e. each lattice site can contain at most one obstacle. The RTP is assumed to have hard-core interactions with obstacles; for the sake of simplicity, its size is, however, supposed negligible in front of the size of the obstacles. The RTP can therefore jump on a lattice site occupied by an obstacle, but cannot cross it. This assumption, illustrated in Supplemental Material (SM), renders the analytical calculations more tractable but does not fundamentally change the phenomenology, as shown in $S M$, where we analyzed the dynamics not allowing jumps of the RTP on occupied sites. We therefore consider the following interaction rule: When the RTP steps on a lattice site occupied by an obstacle, it cannot proceed and effectively gets trapped; the RTP is released by either (i) a tumble leading to a change of polarity or (ii) a step made by the obstacle in any direction. Our goal is to compute analytically the diffusion coefficient $\mathcal{D}=\lim _{t \rightarrow \infty}\left\langle r^{2}(t)\right\rangle /(2 d t)$ as a function of the tumbling probability $\alpha$, the jump probability of the obstacles $\beta$ and the obstacle density $\rho$, where $\langle\ldots\rangle$ represents an average over the trajectories. The dynamics starts with a Poisson distribution of obstacles and we consider timescales much larger than $1 / \alpha$ and $1 / \rho$, so that a stationary state is reached.

First, we decompose the trajectory of the RTP in a sequence of linear runs $\boldsymbol{a}_{i}$, punctuated by either encounters with obstacles, or tumbles in any direction:

$$
\boldsymbol{r}(t)=\sum_{i=1}^{n(t)} \boldsymbol{a}_{i}
$$

with the random variable $n(t)$ being the number of linear runs composing the trajectory up to a given time $t$. Hence, the RTP trajectory is a random sum of random variables and the following exact asymptotics can be obtained by generalizing Wald's identity (see $S M$ ):

$$
\left\langle r^{2}(t)\right\rangle \underset{t \rightarrow \infty}{\sim}\langle n(t)\rangle\left\langle\boldsymbol{a}_{i}^{2}\right\rangle+\sum_{\substack{i, j=1 \\ i \neq j}}^{\langle n(t)\rangle}\left\langle\boldsymbol{a}_{i} \cdot \boldsymbol{a}_{j}\right\rangle .
$$

We now determine explicitly all terms involved in (2). It is useful to decompose a trajectory in successive phases of two types: (i) mobile phases of random duration $\tau_{r}$, when the particle is freely moving on the lattice without interacting with obstacles ; (ii) static phases of random duration $\tau_{s}$ when the particle is trapped by an obstacle. For a trajectory of length $t$, the average number of each of the mobile and static phases is given by $\mathcal{N}_{s}=t /\left(\left\langle\tau_{s}\right\rangle+\left\langle\tau_{r}\right\rangle\right)$. We therefore deduce the mean number of runs performed until time $t$ :

$$
\langle n(t)\rangle \underset{t \rightarrow \infty}{\sim} \frac{t-\mathcal{N}_{s}\left\langle\tau_{s}\right\rangle}{\ell_{p}}=\frac{\left\langle\tau_{r}\right\rangle}{\left(\left\langle\tau_{s}\right\rangle+\left\langle\tau_{r}\right\rangle\right) \ell_{p}} t \equiv \bar{n} t
$$

where the first (persistence length $\ell_{p} \equiv\left\langle\left|\boldsymbol{a}_{i}\right|\right\rangle$ ) and second moment of the run length are given by

$$
\ell_{p}=\frac{1}{1-(1-\rho)(1-\alpha)},\left\langle\boldsymbol{a}_{i}^{2}\right\rangle=\frac{1+(1-\rho)(1-\alpha)}{[1-(1-\rho)(1-\alpha)]^{2}}
$$

In the case of moving obstacles, a trapped particle can be released by two competing independent mechanisms: tumbling of the RTP or stepping of the obstacle. Thus, the mean trapping time reads in the general case:

$$
\left\langle\tau_{s}\right\rangle=\frac{1}{1-\left(1-\alpha^{*}\right)\left(1-\beta^{*}\right)}-1
$$

with probabilities $\alpha^{*}=\alpha(2 d-1) / 2 d$ and $\beta^{*}=\beta(2 d-1) / 2 d$.

Mean run time: Generalized Kac's theorem - We now determine the mean free running time $\left\langle\tau_{r}\right\rangle$. In the case of fixed 
obstacles, it can be exactly defined as the mean return time to the set $\mathcal{O}$ of all obstacles. Remarkably, this quantity can be determined exactly by adapting Kac's theorem [47] (see $S M$ for details). For that purpose we introduce the auxiliary process $\tilde{\boldsymbol{r}}(t)$. It is identical to $\boldsymbol{r}(t)$ in mobile phases, but the durations of all its static phases are set to 1 : Upon each trapping event by an obstacle the auxiliary process is released in the same direction as the original process $\boldsymbol{r}(t)$ would be but after a single time step. The mean running time is then identical for both processes $\boldsymbol{r}(t)$ and $\tilde{\boldsymbol{r}}(t)$; for the process $\tilde{\boldsymbol{r}}(t)$, which has a uniform stationary distribution, the Kac theorem takes a simple form and yields $\left\langle\tau_{r}\right\rangle=1 / P_{\text {stat }}(\mathcal{O})$, where $P_{\text {stat }}(\mathcal{O})$ is the stationary probability of $\mathcal{O}$ for the auxiliary process. We therefore find the simple expression

$$
\left\langle\tau_{r}\right\rangle=\frac{1}{P_{\text {stat }}(\mathcal{O})}=\frac{1}{\rho},
$$

which is strikingly independent of the tumbling probability $\alpha$ and echoes results obtained on continuous space and time processes in confined domains $[49,50]$. Interestingly, this result can be generalized to the case of moving obstacles. To this end, we encode the dynamics of the full system of $N$ obstacles of positions $\mathbf{r}_{i}(t)(1 \leq i \leq N)$ and the auxiliary process $\tilde{\boldsymbol{r}}(t)$ in a $d(N+1)$ tuple $\mathbf{x}(t)$. The process $\mathbf{x}(t)$ performs a symmetric random walk on the hyper cubic lattice of dimension $d(N+1)$, which is, however, not of nearest neighbour type because several particles can move in a given time step. Defining $\mathcal{T}=\left\{\mathbf{x}, \exists i, \mathbf{r}_{i}=\mathbf{r}\right\}$ as the set of trapped configuration, $\left\langle\tau_{r}\right\rangle$ can be defined as the mean return time of the process $\mathbf{x}(t)$ to the set $\mathcal{T}$ and as such verifies $\left\langle\tau_{r}\right\rangle=1 / P_{\text {stat }}(\mathcal{T})$ in virtue of Kac theorem. Here $P_{\text {stat }}(\mathcal{T})$ can depend on the specific choice of microscopic collision rules between the RTP and obstacles. However, it can be generically written $P_{\text {stat }}(\mathcal{T})=C \rho$, where the constant $C$ is of the order 1 and can be exactly set to 1 for a proper choice of microscopic rule [51]. Here, we retain our initial choice, more relevant to real motile systems, and show in $S M$ a very good agreement between our predictions and the results of numerical simulations. This shows finally that, up to a redefinition of microscopic interaction rules, the general expression (6) still holds for moving particles, showing that the mean free running time is universally set by the density of obstacles only, independently of both the tumbling probability of the RTP and the dynamics of obstacles parametrised by $\beta$. This result, key to the derivation below, has potential applications to many other lattice gas models.

Long time correlations - As opposed to the classical RT dynamics in free space, obstacles induce non trivial correlations $\left\langle\boldsymbol{a}_{i} \cdot \boldsymbol{a}_{j}\right\rangle$ that remain to be determined to compute the diffusion coefficient of the RTP [see Eq. (2)]. A first approximation to the diffusion coefficient can be obtained by neglecting these correlations, yielding $\mathcal{D}_{0} \equiv \bar{n}\left\langle\boldsymbol{a}_{i}^{2}\right\rangle /(2 d)$, where $\bar{n}$ and $\left\langle a_{i}^{2}\right\rangle$ are determined exactly by Eqs. (3) and (4). As shown in Fig. 2(b), $\mathcal{D}_{0}$ is already a qualitatively satisfactory approximation. Nevertheless, this approximation fails in the case of mobile obstacles and a more thorough treatment of the correlations is already necessary to obtain exact expressions even in the limit of low density of fixed obstacles. We thus treat exactly the case of fixed obstacles $(\beta=0)$ to lowest order in $\rho$. The correlations can be qualitatively understood in the case of adjacent runs. If $\boldsymbol{a}_{i}$ ends by a trapping event, then clearly $\left\langle\boldsymbol{a}_{i} \cdot \boldsymbol{a}_{i+1}\right\rangle<0$, because the obstacle forbids the run $\boldsymbol{a}_{i+1}$ to keep the direction of $\boldsymbol{a}_{i}$; alternatively if $\boldsymbol{a}_{i}$ ends by a tumble, one still finds $\left\langle\boldsymbol{a}_{i} \cdot \boldsymbol{a}_{i+1}\right\rangle<0$, because particles are less likely to encounter obstacles upon retracing their steps.

More quantitatively, an exact computation confirms this analysis and yields (see $S M$ for details):

$$
\left\langle\boldsymbol{a}_{i} \cdot \boldsymbol{a}_{i+1}\right\rangle=\frac{\alpha}{\alpha+\rho} \frac{\mathcal{C}_{+}-\mathcal{C}_{-}}{2 d}-\frac{\rho}{\alpha+\rho} \frac{\mathcal{C}_{-}}{2 d-1}
$$

with the contribution to positive correlations $\mathcal{C}_{+}=\ell_{p}^{2}$ and to negative correlations $\mathcal{C}_{-}=[1+(1-\rho)(1-\alpha)] /\{[1-$ $\left.(1-\rho)(1-\alpha)]\left[1-(1-\rho)(1-\alpha)^{2}\right]\right\}$. This analysis shows in particular that both contributions of trapping and tumble events yield contributions of order $\mathcal{O}(\rho)$ for $\rho \rightarrow 0$. The exact determination of all correlations $\left\langle\boldsymbol{a}_{i} \cdot \boldsymbol{a}_{j}\right\rangle$ seems out of reach by the direct enumeration techniques used for $j=i+1$; we therefore focus on the small density limit $\rho \rightarrow 0$ and write

$$
\left\langle\boldsymbol{a}_{i} \cdot \boldsymbol{a}_{i+k}\right\rangle \underset{\rho \rightarrow 0}{=} g(\alpha, k) \frac{\rho}{\alpha}+o(\rho),
$$

where a generalization of the argument given above for $k=$ 1 shows that $\forall k, g(\alpha, k) \neq 0$. The only two lengthscales entering this problem are $1 / \rho$ and $1 / \alpha$; for all $k, g(\alpha, k)$ has the dimension of a length squared. In the limit of low density, the only relevant lengthscale left is $1 / \alpha$, and a dimensional analysis yields (taking $\alpha$ small) $g(\alpha, k) \underset{\alpha \rightarrow 0}{\sim}-\xi_{k} / \alpha^{2}$, where $\xi_{k}$ is a lattice-dependent dimensionless number; for $k=1 \mathrm{Eq}$. (7) yields the exact value $\xi_{1}=11 / 24$.

Finally, the correction to the diffusion coefficient reads

$$
\frac{\mathcal{D}-\mathcal{D}_{0}}{\bar{n} / 2 d} \underset{\rho, \alpha \rightarrow 0}{\sim}-\frac{2 \rho}{\alpha^{3}} \sum_{k=1}^{\infty} \xi_{k} .
$$

This result is exact to linear order in $\rho$ in the limit $\alpha \rightarrow 0$. It involves dimensionless constants $\xi_{k}$, which are found numerically to satisfy $\xi_{k} \approx \xi_{1} \Gamma^{k-1}$, with $\Gamma \approx 0.22$. We show on Fig. 2(a) a perfect agreement between our numerical simulations and Eq. (9).

We now aim at obtaining an approximate solution uniformly accurate in $\alpha$; to this end, we need to go beyond the linear order in $\rho / \alpha$ and therefore consider non vanishing correlations $\left\langle\boldsymbol{a}_{i} \cdot \boldsymbol{a}_{i+1}\right\rangle$. We also generalize our argument here to mobile obstacles, for which the nearest neighbor correlations need to be amended. Indeed, two independent mechanisms can now release the RTP when trapped by an obstacle, yielding correlations of a different nature: (i) a tumble of the RTP, as in the case of fixed obstacles, or (ii) a step made by the obstacle away from the course of the RTP. The latter induces large correlations in the limit $\alpha \rightarrow 0$ which must be taken into account to quantitatvely describe the RTP dynamics. Taking 

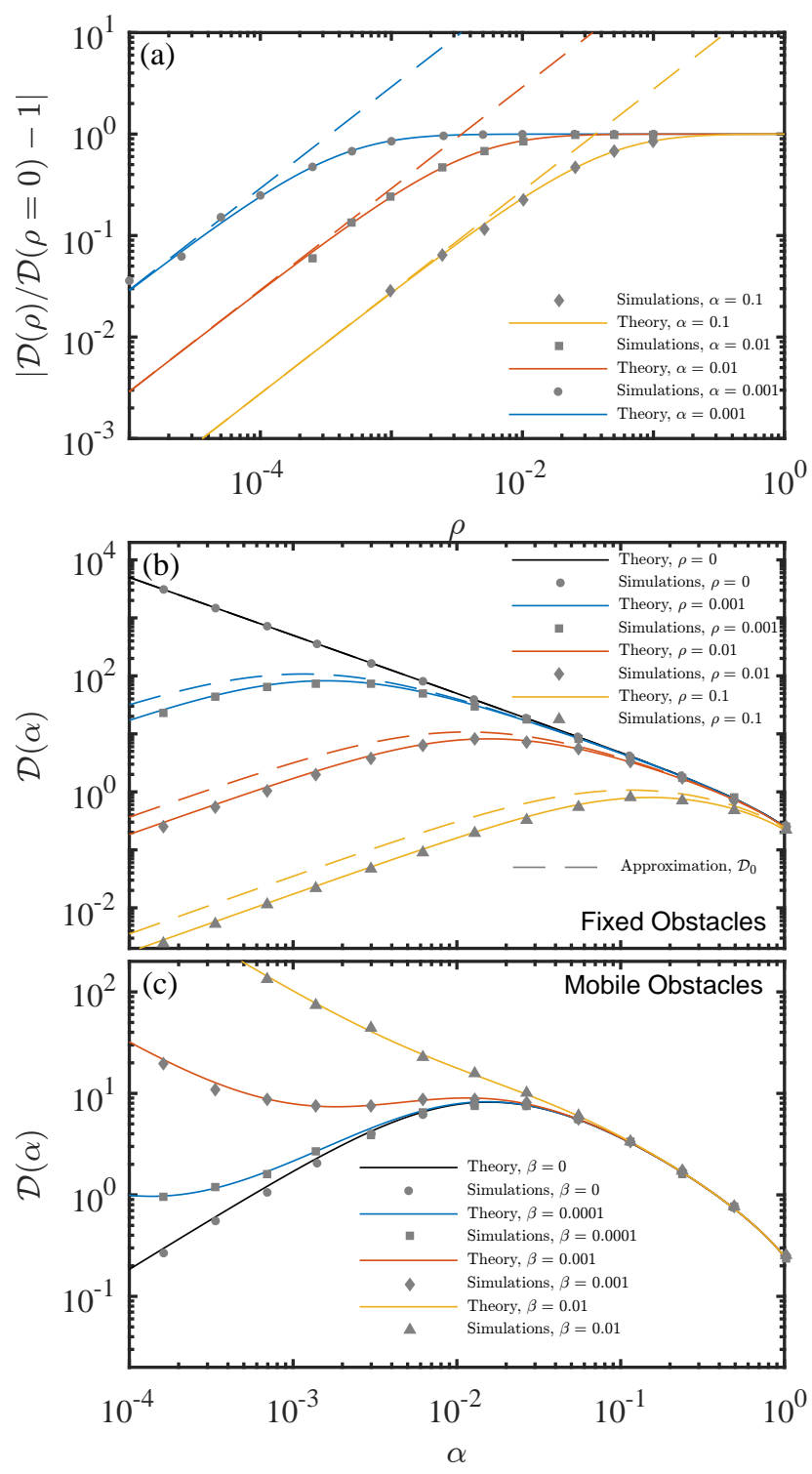

FIG. 2. Diffusivity of a run-and-tumble particle - (a) Diffusion coefficients for fixed obstacles relative to the free diffusion coefficient $\mathcal{D}(\rho=0)=(2-\alpha) / \alpha$ at various tumbling rates $\alpha$ as a function of the obstacles density $\rho$, dashed lines show the linear expansion in $\rho$. Diffusion coefficients for (b) fixed obstacles at various obstacle densities $\rho$ and (c) mobile obstacles at various obstacles mobilities $\beta$ and density $\rho=0.01$ as a function of the tumbling probability $\alpha$. In both cases, the symbols are results of numerical simulations and the solid lines are given by Eq. 11 . In (b), dashed lines show $\mathcal{D}_{0}$, our approximation where correlations between runs are neglected.

these events into account yields

$$
\begin{aligned}
\left\langle\boldsymbol{a}_{i} \cdot \boldsymbol{a}_{i+1}\right\rangle= & \frac{\alpha}{\alpha+\rho} \frac{\mathcal{C}_{+}-\mathcal{C}_{-}}{2 d}+\frac{\rho}{\alpha+\rho}\left(\frac{\beta^{*}}{\alpha^{*}+\beta^{*}} \mathcal{C}_{+}-\right. \\
& \left.\frac{\alpha^{*}}{\alpha^{*}+\beta^{*}} \frac{\mathcal{C}_{-}}{2 d-1}\right) \equiv \gamma \ell_{p}^{2}
\end{aligned}
$$

which yields the exact Eq. (7) in the limit of fixed obstacles, $\beta \rightarrow 0$. In order to cover the regime of large correlations, we next assume that correlations are induced by interactions between successive runs only; classical results [52] then yield $\left\langle\boldsymbol{a}_{i} \cdot \boldsymbol{a}_{j}\right\rangle=\gamma^{|i-j|} \ell_{p}^{2}$. We have checked numerically that these correlations decay exponentially (see $S M$ ). After summation, we obtain finally

$$
\mathcal{D} \underset{\rho \rightarrow 0}{\sim} \frac{\bar{n}}{2 d}\left[\left\langle\boldsymbol{a}_{i}^{2}\right\rangle+\frac{2 \gamma}{1-\gamma} \ell_{p}^{2}\right]
$$

where $\bar{n},\left\langle\boldsymbol{a}_{i}^{2}\right\rangle, \ell_{p}, \gamma$ are defined explicitly in Eqs. (3),(4), and (10). This explicit expression, though approximate, provides a uniformly accurate determination of the diffusion coefficient as we show below; it is in addition consistent with the exact limit $(\alpha, \beta, \rho \rightarrow 0)$ defined above.

Optimized diffusivity of the RTP - In Fig. 2, we show our theoretical predictions for the diffusion coefficient in the case of static and mobile obstacles along with the results of simulations. We observe a very good agreement between theory and simulations. For fixed obstacles $(\beta=0)$, the diffusion coefficient is nonmonotonic in the tumbling probability $\alpha$. Qualitatively, this behavior can be understood as follows: (i) in the limit of high tumbling probability $\alpha \sim 1$, the RTP tumbles at each time step; decreasing $\alpha$ then increases the long time diffusion coefficient by increasing the persistence length; (ii) in the limit of low tumbling probability $\alpha \rightarrow 0$, the RTP proceeds mainly in long straight runs, interrupted by trapping events whose duration $\tau_{s}$ diverges for $\alpha \rightarrow 0$ leading to a vanishing diffusion coefficient. The analysis of Eq. (11) shows that the optimal tumbling probability satisfies $\alpha_{m} \propto \rho$ ; in turn, the optimal diffusion coefficient is found to scale as $\mathcal{D}_{m} \propto 1 / \rho$.

In the case of mobile obstacles, the non-monotonicity in the diffusion coefficient is preserved only for a low enough obstacle mobility $\beta \leq \beta_{c} \propto \rho$. While the diffusion coefficient in the limit of high tumbling probability is independent of the obstacle mobility in the regime $\rho \rightarrow 0$, not surprisingly, it shows a strong dependence on $\beta$ at low tumbling probability. It is interesting to note that no matter the obstacle mobility the RTP diffusion coefficient always monotonically increases for low enough decreasing tumbling probability $(\alpha \lesssim \beta)$ showing that in these cases, the important unlocking mechanism is obstacle mobility.

Discussion - Using a minimal model of RTPs in crowded environments, we have shown that such active particles display a nonmonotonic diffusivity as a function of the tumbling probability for static and mobile obstacles. Our analytical prediction is exact in the limit of low obstacle density for fixed obstacles. Its derivation is based on the generalization of a theorem by Kac, a strikingly simple result expected to find a variety of applications in general lattice gas problems. While derived for a particular model for which analytical progress was tractable, our results qualitatively extend far beyond this case. First, we show in $S M$ that they extend to other microscopic types of obstacles. Then, a similar behavior has been 
previously observed in a mean-field model of bacterial diffusion in porous media [53]; our result is also reminiscent of the negative differential mobility observed in Refs. [40, 44] for active tracer particles. Furthermore, our results, which are exact in the low density limit, extend qualitatively to moderate to high densities of relevance to experimental settings and in particular to the diffusion of bacteria in soft agar gels [53, 54]. Finally, while the derivation of the results reported in this Letter is specific to RTPs on lattice, the underlying mechanisms are not, and we expect similarly rich behaviors for the diffusivities of other active particles, on lattice or in continuous space. This could potentially lead to an optimization of the diffusion coefficient of active particles with respect to their reorientation dynamics and, as such, to an enhancement of their transport properties or exploration efficiency in crowded environment.

We acknowledge financial support from Institut National du Cancer (T.B. and R.V.) and from Agence Nationale de la Recherche through the grant Bacttern (Y.Z. and J.T.).

* Electronic address: thibault.bertrand@upmc.fr

$\dagger$ Electronic address: voiturie@1ptmc.jussieu.fr

[1] S. Ramaswamy, Annual Review of Condensed Matter Physics 1, 323 (2010).

[2] C. Bechinger, R. Di Leonardo, H. Löwen, C. Reichhardt, G. Volpe, and G. Volpe, Rev. Mod. Phys. 88, 045006 (2016).

[3] M. J. Schnitzer, Phys. Rev. E 48, 2553 (1993).

[4] H. C. Berg, E. coli in Motion, 1st ed. (Springer-Verlag New York, 2004).

[5] R. Di Leonardo, L. Angelani, D. Dell'Arciprete, G. Ruocco, V. Iebba, S. Schippa, M. P. Conte, F. Mecarini, F. De Angelis, and E. Di Fabrizio, Proceedings of the National Academy of Sciences 107, 9541 (2010).

[6] J. Saragosti, V. Calvez, N. Bournaveas, B. Perthame, A. Buguin, and P. Silberzan, Proceedings of the National Academy of Sciences 108, 16235 (2011).

[7] J. Schwarz-Linek, C. Valeriani, A. Cacciuto, M. E. Cates, D. Marenduzzo, A. N. Morozov, and W. C. K. Poon, Proceedings of the National Academy of Sciences 109, 4052 (2012).

[8] M. Polin, I. Tuval, K. Drescher, J. P. Gollub, and R. E. Goldstein, Science 325, 487 (2009).

[9] M. L. Heuzé, P. Vargas, M. Chabaud, M. Le Berre, Y.-J. Liu, O. Collin, P. Solanes, R. Voituriez, M. Piel, and A.-M. LennonDuménil, Immunol Rev 256, 240 (2013).

[10] O. Bénichou, C. Loverdo, M. Moreau, and R. Voituriez, Rev. Mod. Phys. 83, 81 (2011).

[11] T. Vicsek, A. Czirók, E. Ben-Jacob, I. Cohen, and O. Shochet, Phys. Rev. Lett. 75, 1226 (1995).

[12] J. Deseigne, O. Dauchot, and H. Chaté, Phys. Rev. Lett. 105, 098001 (2010).

[13] A. Bricard, J.-B. Caussin, N. Desreumaux, O. Dauchot, and D. Bartolo, Nature 503, 95 (2013).

[14] A. P. Solon, H. Chaté, and J. Tailleur, Phys. Rev. Lett. 114, 068101 (2015).

[15] I. Theurkauff, C. Cottin-Bizonne, J. Palacci, C. Ybert, and L. Bocquet, Phys. Rev. Lett. 108, 268303 (2012).

[16] I. Buttinoni, J. Bialké, F. Kümmel, H. Löwen, C. Bechinger, and T. Speck, Phys. Rev. Lett. 110, 238301 (2013).
[17] J. Palacci, S. Sacanna, A. P. Steinberg, D. J. Pine, and P. M. Chaikin, Science 339, 936 (2013).

[18] J. Tailleur and M. E. Cates, Phys. Rev. Lett. 100, 218103 (2008).

[19] Y. Fily and M. C. Marchetti, Phys. Rev. Lett. 108, 235702 (2012).

[20] G. S. Redner, M. F. Hagan, and A. Baskaran, Phys. Rev. Lett. 110, 055701 (2013).

[21] E. Pinçe, S. K. P. Velu, A. Callegari, P. Elahi, S. Gigan, G. Volpe, and G. Volpe, Nature Communications 7, 10907 EP (2016).

[22] G. Volpe and G. Volpe, Proceedings of the National Academy of Sciences 114, 11350 (2017), http://www.pnas.org/content/114/43/11350.full.pdf.

[23] M. Zeitz, K. Wolff, and H. Stark, The European Physical Journal E 40, 23 (2017).

[24] C. Sándor, A. Libál, C. Reichhardt, and C. J. Olson Reichhardt, Phys. Rev. E 95, 032606 (2017).

[25] C. Reichhardt and C. J. O. Reichhardt, Phys. Rev. E 91, 032313 (2015).

[26] A. P. Berke, L. Turner, H. C. Berg, and E. Lauga, Phys. Rev. Lett. 101, 038102 (2008).

[27] H. H. Wensink and H. Löwen, Phys. Rev. E 78, 031409 (2008).

[28] J. Elgeti and G. Gompper, EPL (Europhysics Letters) 85, 38002 (2009).

[29] J. Elgeti and G. Gompper, EPL (Europhysics Letters) 101, 48003 (2013).

[30] I. D. Vladescu, E. J. Marsden, J. Schwarz-Linek, V. A. Martinez, J. Arlt, A. N. Morozov, D. Marenduzzo, M. E. Cates, and W. C. K. Poon, Phys. Rev. Lett. 113, 268101 (2014).

[31] A. Bricard, J.-B. Caussin, D. Das, C. Savoie, V. Chikkadi, K. Shitara, O. Chepizhko, F. Peruani, D. Saintillan, and D. Bartolo, Nature Communications 6, 7470 EP (2015).

[32] O. Chepizhko and F. Peruani, Phys. Rev. Lett. 111, 160604 (2013).

[33] J. Tailleur and M. Cates, EPL (Europhysics Letters) 86, 60002 (2009).

[34] A. Kaiser, H. H. Wensink, and H. Löwen, Phys. Rev. Lett. 108, 268307 (2012).

[35] A. Kaiser, K. Popowa, H. H. Wensink, and H. Löwen, Phys. Rev. E 88, 022311 (2013).

[36] P. Galajda, J. Keymer, P. Chaikin, and R. Austin, Journal of Bacteriology 189, 8704 (2007).

[37] A. Guidobaldi, Y. Jeyaram, I. Berdakin, V. V. Moshchalkov, C. A. Condat, V. I. Marconi, L. Giojalas, and A. V. Silhanek, Phys. Rev. E 89, 032720 (2014).

[38] L. Restrepo-Perez, L. Soler, C. S. Martinez-Cisneros, S. Sanchez, and O. G. Schmidt, Lab Chip 14, 1515 (2014).

[39] C. Reichhardt and C. J. Olson Reichhardt, Phys. Rev. E 90, 012701 (2014).

[40] C. Reichhardt and C. J. O. Reichhardt, Journal of Physics: Condensed Matter 30, 015404 (2018).

[41] A. Morin, N. Desreumaux, J.-B. Caussin, and D. Bartolo, Nature Physics 13, 63 (2017).

[42] K. Nakazato and K. Kitahara, Progress of Theoretical Physics 64, 2261 (1980).

[43] O. Bénichou, A. Bodrova, D. Chakraborty, P. Illien, A. Law, C. Mejía-Monasterio, G. Oshanin, and R. Voituriez, Phys. Rev. Lett. 111, 260601 (2013).

[44] O. Bénichou, P. Illien, G. Oshanin, A. Sarracino, and R. Voituriez, Phys. Rev. Lett. 113, 268002 (2014).

[45] S. Leitmann and T. Franosch, Phys. Rev. Lett. 111, 190603 (2013).

[46] J. Cividini, D. Mukamel, and H. A. Posch, Phys. Rev. E 95, 012110 (2017). 
[47] D. Aldous and J. A. Fill, "Reversible markov chains and random walks on graphs," (2002), unfinished monograph, recompiled 2014, available at http://www.stat.berkeley.edu/ \$ \sim\$aldous/RWG/book.html.

[48] S. Condamin, O. Bénichou, and M. Moreau, Phys. Rev. E 72, 016127 (2005).

[49] O. Bénichou, M. Coppey, M. Moreau, P. H. Suet, and R. Voituriez, EPL (Europhysics Letters) 70, 42 (2005).

[50] S. Blanco and R. Fournier, EPL (Europhysics Letters) 61, 168
(2003).

[51] i.e., the choice of the order in the sequence of particle tumbles and particle moves during a given time step.

[52] B. D. Hughes, Random Walks and Random Environments: Random walks, Oxford Science Publications, Vol. 1 (Clarendon Press, New York, 1995).

[53] N. A. Licata, B. Mohari, C. Fuqua, and S. Setayeshgar, Biophysical Journal 110, 247 (2016).

[54] O. A. Croze, G. P. Ferguson, M. E. Cates, and W. C. Poon, Biophysical Journal 101, 525 (2011). 


\section{Supplemental Material for "Optimized Diffusion of Run-and-Tumble Particles in Crowded Environments"}

\section{SCHEMATIC DESCRIPTION OF AN OBSTACLE}

In this section, we illustrate on Figure S1 the definition of the obstacles we consider in the main text. We consider obstacles (in light grey) interacting via hard-core repulsion, i.e. each lattice site can contain at most one obstacle. The run-and-tumble particle (RTP) is assumed to have hard-core interactions with obstacles; we consider its size to be negligible in front of the size of the obstacles. The RTP can therefore jump on a lattice site occupied by an obstacle. When a RTP (black dot with polarity along the black arrow) arrives on a site (i,j) occupied by an obstacle, the latter prevents the RTP from going onwards along its polarity. The RTP only resumes its motion after either: (i) a tumble leading to one of the escape routes highlighted by the light blue dashed arrows or (ii) if the obstacles diffuses to another lattice site.

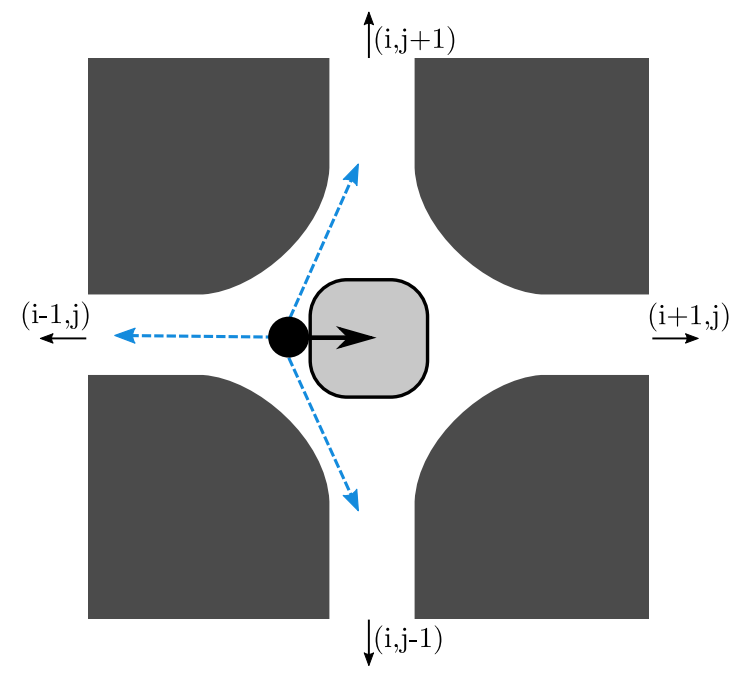

FIG. S1. Schematic description of the obstacles in our lattice gas model

\section{GENERALIZATION OF WALD'S IDENTITY AND EQUATION (2) OF MAIN TEXT}

We start from the decomposition of the trajectories of the RTP in a sequence of linear runs $\boldsymbol{a}_{i}$, punctuated by either encounters with obstacles, or tumbles in any direction, as defined in the main text:

$$
\boldsymbol{r}(t)=\sum_{i=1}^{n(t)} \boldsymbol{a}_{i}
$$

Here the random variable $n(t)$ denotes the number of linear runs composing the trajectory up to a given time $t$. We introduce the probability $p_{t}(n)$ that $n$ linear runs occurred up to time $t$. We then write

$$
\left\langle r^{2}(t)\right\rangle=\sum_{n=0}^{+\infty}\left\langle r^{2}(t)\right\rangle_{n} p_{t}(n)
$$

where $\left\langle r^{2}(t)\right\rangle_{n}$ denotes the average conditioned by $n$. It follows that

$$
\left\langle r^{2}(t)\right\rangle=\langle n(t)\rangle\left\langle\boldsymbol{a}_{i}^{2}\right\rangle+\sum_{n=0}^{+\infty} p_{t}(n) \sum_{\substack{i, j=1 \\ i \neq j}}^{n}\left\langle\boldsymbol{a}_{i} \cdot \boldsymbol{a}_{j}\right\rangle .
$$


Then one has

$$
\left|\sum_{n=0}^{+\infty} p_{t}(n) \sum_{\substack{i, j=1 \\ i \neq j}}^{n}\left\langle\boldsymbol{a}_{i} \cdot \boldsymbol{a}_{j}\right\rangle-\sum_{\substack{i, j=1 \\ i \neq j}}^{\langle n\rangle}\left\langle\boldsymbol{a}_{i} \cdot \boldsymbol{a}_{j}\right\rangle\right| \leq\left\langle\boldsymbol{a}_{i}^{2}\right\rangle\langle|n-\langle n\rangle|\rangle \underset{t \rightarrow \infty}{\ll}\langle n(t)\rangle\left\langle\boldsymbol{a}_{i}^{2}\right\rangle .
$$

Here we have used the fact that $\langle n(t)\rangle \underset{t \rightarrow \infty}{\sim} \bar{n} t$, as justified in the main text, and $\sqrt{\left\langle(n(t)-\langle n(t)\rangle)^{2}\right\rangle} /\langle n(t)\rangle \rightarrow \underset{t \rightarrow \infty}{\rightarrow} 0$. Finally, this yields Equation (2) of the main text:

$$
\left\langle r^{2}(t)\right\rangle \underset{t \rightarrow \infty}{\sim}\langle n(t)\rangle\left\langle\boldsymbol{a}_{i}^{2}\right\rangle+\sum_{\substack{i, j=1 \\ i \neq j}}^{\langle n(t)\rangle}\left\langle\boldsymbol{a}_{i} \cdot \boldsymbol{a}_{j}\right\rangle
$$

\section{APPLICATION OF KAC'S THEOREM ON THE MEAN RETURN TIME OF MARKOV PROCESSES}

The general statement of Kac theorem for the mean return time of Markov chain can be found in [47]. This theorem takes a simple form in the case of irreducible finite state discrete time Markov processes $\left(X_{t}\right)$, if one considers a subset $A$ of states of interest such that $X_{t} \in A \rightarrow X_{t+1} \notin A$ : the process $X_{t}$ spends a single time step in $A$ at each visit of $A$. The Kac theorem then gives that the mean of the return time $T_{r}$ defined by $T_{r}=\inf \left\{t \geq 1: X_{0} \in A, X_{t} \in A\right\}$ is exactly given by

$$
\left\langle T_{t}\right\rangle=1 / P_{s}(A)
$$

where $P_{s}(A)$ is the stationary measure of the set $A$. In the case of the RT dynamics with obstacles that we study in this paper, in order to apply Kac theorem in this simple form, we had to introduce the auxiliary process $\tilde{\boldsymbol{r}}(t)$. It is identical to $\boldsymbol{r}(t)$ in mobile phases, but the durations of all its static phases are set to 1 : upon each trapping event by an obstacle the auxiliary process is released in the same direction as the original process $\boldsymbol{r}(t)$ would be, but after a single time step. The mean running time is then identical for both the original process of interest $\boldsymbol{r}(t)$ and $\tilde{\boldsymbol{r}}(t)$; for the process $\tilde{\boldsymbol{r}}(t)$, which has a uniform stationary distribution, Kac theorem is directly applicable and yields $\left\langle\tau_{r}\right\rangle=1 / P_{\text {stat }}(\mathcal{O})$, where $P_{\text {stat }}(\mathcal{O})$ is the stationary probability of $\mathcal{O}$ for the auxiliary process. We therefore find the simple expression

$$
\left\langle\tau_{r}\right\rangle=\frac{1}{P_{\text {stat }}(\mathcal{O})}=\frac{1}{\rho}
$$

which is strikingly independent of the tumbling probability $\alpha$. It should be noted that the position process $r(t)$ is not strictly Markovian, because the polarity of the particle needs to be specified to obtain a Markovian evolution. The process $\boldsymbol{r}(t)$ being nevertheless ergodic Kac theorem still applies.

\section{DERIVATION OF THE NEAREST NEIGHBOR CORRELATIONS}

In this section, we provide additional details about the derivation of the nearest neighbor correlations $\left\langle\boldsymbol{a}_{i} \cdot \boldsymbol{a}_{i+1}\right\rangle$. We only consider here the case of fixed obstacles. In particular, in steady state, every site has the same occupation probability $\rho$ and the two mechanisms terminating a run (tumble or encounter with an obstacle) are statistically independent (this problem can be seen as an infinite sequence of Bernoulli trials); we can write the effective persistence length as follows

$$
\ell_{p} \equiv\left\langle\left|\boldsymbol{a}_{i}\right|\right\rangle=\sum_{n=1}^{\infty} n(1-\rho)^{n-1}(1-\alpha)^{n-1}[1-(1-\rho)(1-\alpha)]=\frac{1}{1-(1-\rho)(1-\alpha)}
$$

As in the main text, we can partition these correlations between the two types of events ending straight run $i$ : (1) a tumble and (2) an encounter with an obstacle followed by an unlocking tumble. Thus, we write

$$
\left\langle\boldsymbol{a}_{i} \cdot \boldsymbol{a}_{i+1}\right\rangle=w_{t}\left\langle\boldsymbol{a}_{i} \cdot \boldsymbol{a}_{i+1}\right\rangle_{t}+w_{o}\left\langle\boldsymbol{a}_{i} \cdot \boldsymbol{a}_{i+1}\right\rangle_{o}
$$




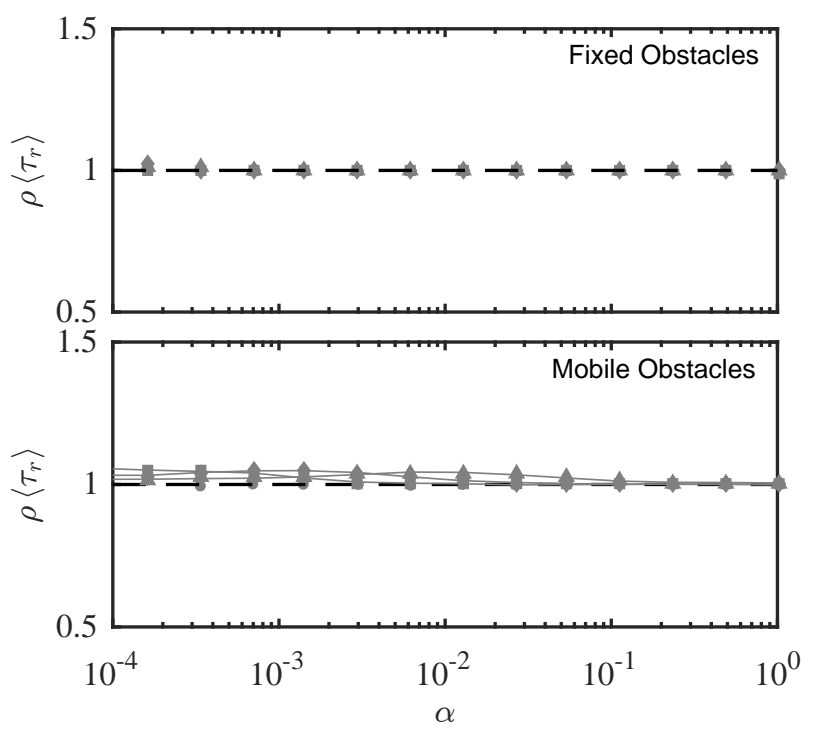

FIG. S2. Running times - Average running time $\left\langle\tau_{R}\right\rangle$ on a semilog scale renormalized by Kac's prediction $1 / \rho$ for: (Top) Fixed obstacles $(\beta=0)$ at various densities $\rho=10^{-3}$ (squares), $10^{-2}$ (diamonds) and $10^{-1}$ (triangles); (Bottom) Mobile obstacles at fixed density $\rho=10^{-2}$ for various mobilities $\beta=0$ (circles), $10^{-4}$ (squares), $10^{-3}$ (diamonds) and $10^{-2}$ (triangles). The dashed lines are the theoretical predictions.

with $w_{t}$ and $w_{o}$ the probabilities that the run ended in a tumble and an encounter with an obstacle respectively. By definition, these probabilities read

$$
\begin{aligned}
w_{t} & \equiv \frac{\alpha}{\alpha+\rho} \\
w_{o} & \equiv \frac{\rho}{\alpha+\rho}
\end{aligned}
$$

On one hand, a straight run ending in an encounter with an obstacle (followed by an unlocking tumble) can only lead to negative correlations, as the following run needs to be polarized in the exact opposite direction to have a non-zero contribution. Indeed, orthogonal directions would lead to null contributions and a tumble ending in the TP polarized in the original direction would not be unlocking (only $2 d-1$ directions are unlocking the TP). Hence, we can write

$$
\left\langle\boldsymbol{a}_{i} \cdot \boldsymbol{a}_{i+1}\right\rangle_{o}=\frac{-\mathcal{C}_{-}}{2 d-1}
$$

On the other hand, a straight run ending in a tumble (with no obstacle) will lead to both positive and negative contributions. Indeed, a tumble can lead to a non-zero contribution by polarizing the TP either in the original polarization (positive correlations) or in the opposite direction to the original polarization (negative correlations) with same probability $1 /(2 d)$, any other direction of the new polarization will lead to zero contribution to the correlations. As such, we write

$$
\left\langle\boldsymbol{a}_{i} \cdot \boldsymbol{a}_{i+1}\right\rangle_{t}=\frac{\mathcal{C}_{+}-\mathcal{C}_{-}}{2 d}
$$

First, we consider the positive correlations between runs $i$ and $i+1$. The fundamental process we are modelling here is Markovian, as such two following runs are independent and as a consequence, we can write

$$
\mathcal{C}_{+}=\left\langle\left|\boldsymbol{a}_{i}\right|\right\rangle\left\langle\left|\boldsymbol{a}_{i+1}\right|\right\rangle=\left\langle\left|\boldsymbol{a}_{i}\right|\right\rangle^{2}=\ell_{p}^{2}=\frac{1}{[1-(1-\rho)(1-\alpha)]^{2}}
$$

Let us now turn to the negative correlations which require a bit more work. Indeed, negative correlations come from runs in opposite directions. Qualitatively, for all steps of the run $i+1$ overlapping with run $i$, the TP will not encounter any obstacle with probability 1 and are therefore favored. Here, we partition the correlations as a function of the event starting the run $i$ and 

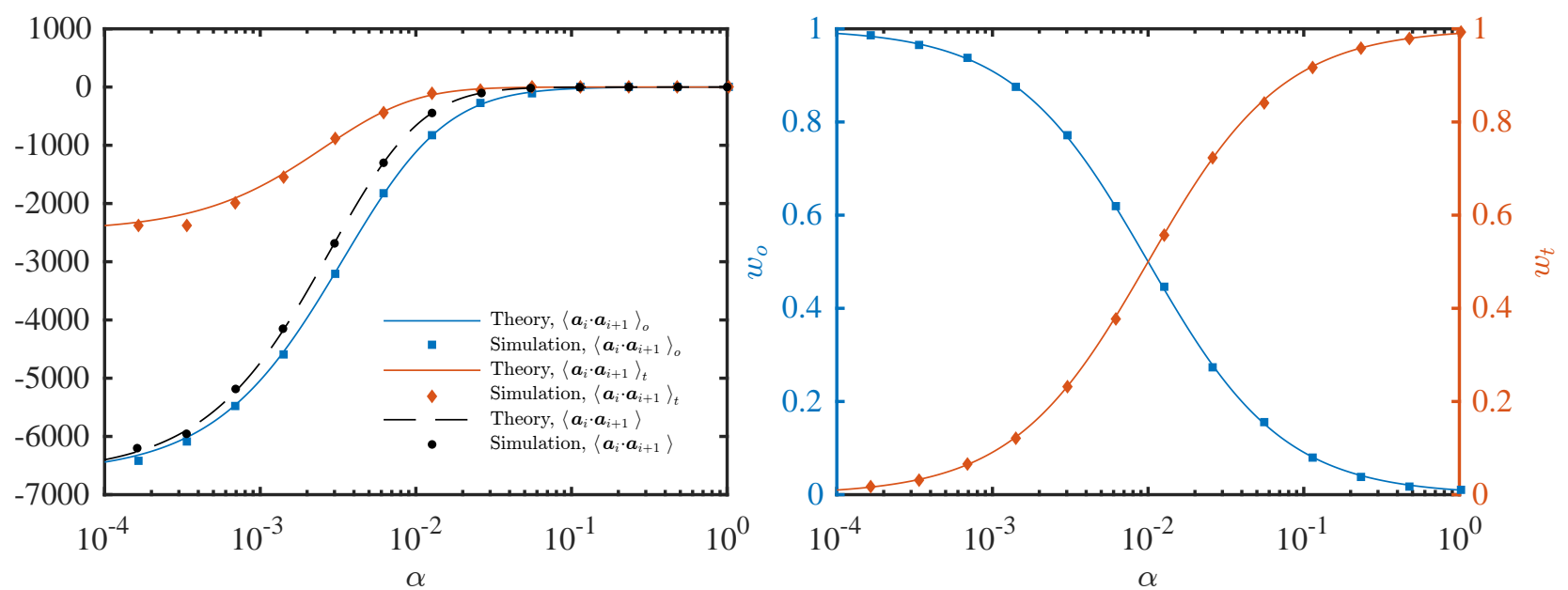

FIG. S3. Nearest neighbor correlations - (Left) Decomposition of all terms in correlations between runs $i$ and $i+1$ from Equations S12, S13 and S9 (lines) and from numerical simulations (symbols - averaged over long runs for at least 1000 different obstacle configurations) displayed as a function of the tumbling probability $\alpha$; (Right) Weight of each type of correlations (coming from a simple tumble or an encounter with an obstacle) from their definition (solid lines) and as measured in numerical simulations (symbols). All data and theoretical predictions obtained for $\rho=0.01$.

we write

$$
\begin{aligned}
\mathcal{C}_{-}= & \sum_{n=1}^{\infty} n(1-\rho)^{n-1}(1-\alpha)^{n-1} \rho\left[\sum_{m=1}^{n-1} m(1-\alpha)^{m-1} \alpha+n(1-\alpha)^{n-1}\right]+ \\
& \sum_{n=1}^{\infty} n(1-\rho)^{n-1}(1-\alpha)^{n-1} \alpha(1-\rho)\left[\sum_{m=1}^{n} m(1-\alpha)^{m-1} \alpha\right. \\
& \left.+\sum_{m=n+1}^{\infty} m(1-\alpha)^{m-1}(1-\rho)^{m-n-1}[1-(1-\rho)(1-\alpha)]\right]
\end{aligned}
$$

The first term in Equation S15 corresponds to the runs $i$ starting by unlocking from an obstacle. In this case, we know that the run $i+1$ can be at most as long as run $i$. We also know that, for the whole length of run $i+1$, the TP will not encounter any obstacle and the only possible event is a tumble of the RTP. The second term corresponds to trajectories with run $i$ starting with a tumble (and no obstacle). Here, when the run $i$ is of length $n$, we know with certainty that the TP will not encounter any obstacle for its first $n$ steps in run $i+1$. In this case, run $i+1$ is not limited to be at most as long as run $i$ and can thus exceed the length of run $i$; as a consequence, for runs $i$ of length $n$, we partition the correlations over runs $i+1$ of length $m \leq n$ and runs $i+1$ of length $m \geq n+1$ and give them proper weights. One can easily check that these conditional probabilities are properly normalized. Finally, Equation S15 reduces to

$$
\mathcal{C}_{-}=\frac{1+(1-\rho)(1-\alpha)}{[1-(1-\rho)(1-\alpha)]\left[1-(1-\rho)(1-\alpha)^{2}\right]}
$$

Figure S3 shows a perfect agreement between the nearest neighbor correlation terms as calculated from our theoretical prediction and measured in numerical simulations. Further, we find that

$$
\lim _{\rho \rightarrow 0} \mathcal{C}_{-}=\frac{1}{\alpha^{2}}
$$

which is consistent with the fact that when $\rho \rightarrow 0$, the successive runs are all independent of each other and thus $\left\langle\boldsymbol{a}_{i} \cdot \boldsymbol{a}_{i+1}\right\rangle \underset{\rho \rightarrow 0}{\rightarrow}$ 0 . In the limit of low tumbling probability (for finite density), we find unsurprisingly that only the negative correlations term matters and we have

$$
\lim _{\alpha \rightarrow 0}\left\langle\boldsymbol{a}_{i} \cdot \boldsymbol{a}_{i+1}\right\rangle=\frac{1}{2 d-1} \frac{\rho-2}{\rho^{2}}
$$


To go one step further, we expand the nearest neighbor correlations in power series of the density $\rho$ and we obtain

$$
\left\langle\boldsymbol{a}_{i} \cdot \boldsymbol{a}_{i+1}\right\rangle \underset{\rho \rightarrow 0}{=} g(\alpha, 1) \frac{\rho}{\alpha}+o(\rho)
$$

with

$$
g(\alpha, 1)=\frac{3 \alpha^{2}-10 \alpha+11}{12 \alpha^{2}(\alpha-2)}
$$

Finally, we find by taking first the limit of low density $(\rho \rightarrow 0)$ and next the limit of low tumbling probability $(\alpha \rightarrow 0)$, always keeping $\rho \ll \alpha$, the correlations between successive runs read

$$
\left\langle\boldsymbol{a}_{i} \cdot \boldsymbol{a}_{i+1}\right\rangle \underset{\rho, \alpha \rightarrow 0}{=}-\frac{\xi_{1}}{\alpha^{2}} \frac{\rho}{\alpha}+o(\rho)
$$

with $\xi_{1}=11 / 24$. This result is consistent with the fact that $\rho / \alpha$ is the only non-dimensional parameter in this problem and that in the limit $\rho \rightarrow 0$ (free diffusion), the only lengthscale in the system is $1 / \alpha$. By dimensional analysis, one obtains that the correlations between successive runs must scale as $\rho / \alpha^{3}$.

\section{DERIVATION OF THE DIFFUSION COEFFICIENT FOR FIXED OBSTACLES}

The above argument can be generalized to higher order correlations, even though an explicit calculation seems out of reach. We find that higher order correlations are also linear in the lowest order of the obstacle density

$$
\left\langle\boldsymbol{a}_{i} \cdot \boldsymbol{a}_{i+k}\right\rangle \underset{\rho \rightarrow 0}{=} g(\alpha, k) \frac{\rho}{\alpha}+o(\rho)
$$

In the limit of low tumbling probabilities (with $\rho \ll \alpha$ ), we found that $g(\alpha, k) \underset{\alpha \rightarrow 0}{\sim}-\xi_{k} / \alpha^{2}$. The diffusion coefficient is given by

$$
\mathcal{D} \equiv \lim _{t \rightarrow \infty} \frac{\left\langle r^{2}(t)\right\rangle}{2 d t}
$$

with $\left\langle r^{2}(t)\right\rangle \underset{t \rightarrow \infty}{\sim}\langle n(t)\rangle\left\langle\boldsymbol{a}_{i}^{2}\right\rangle+\sum_{i, j=1}^{\langle n(t)\rangle}\left\langle\boldsymbol{a}_{i} \cdot \boldsymbol{a}_{j}\right\rangle$. In the long time limit, we can rewrite this

$$
\left\langle r^{2}(t)\right\rangle \underset{t \rightarrow \infty}{\sim}\langle n(t)\rangle\left[\left\langle\boldsymbol{a}_{i}^{2}\right\rangle+2 \sum_{k=1}^{\infty}\left\langle\boldsymbol{a}_{i} \cdot \boldsymbol{a}_{i+k}\right\rangle\right] .
$$

We can sum over all possible correlations and the diffusion coefficient thus reads

$$
\mathcal{D} \underset{\rho, \alpha \rightarrow 0}{\sim} \frac{\bar{n}}{2 d}\left[\left\langle\boldsymbol{a}_{i}^{2}\right\rangle-\frac{2}{\alpha^{2}} \frac{\rho}{\alpha} \sum_{k=1}^{\infty} \xi_{k}\right]
$$

with $\left\langle a_{i}^{2}\right\rangle=[1+(1-\rho)(1-\alpha)] /[1-(1-\rho)(1-\alpha)]^{2}$. This result is exact in the limit $\rho \rightarrow 0$ for static obstacles. In practice, we find a good approximation in $\xi_{k}=\xi_{1} \Gamma^{k-1}$ when $\alpha \rightarrow 0$. Finally, in the limit of $\rho \rightarrow 0, \alpha \rightarrow 0$ with $\rho<<\alpha$, the diffusion coefficient reads

$$
\mathcal{D} \approx \frac{\bar{n}}{2 d}\left[\left\langle\boldsymbol{a}_{i}^{2}\right\rangle-\frac{2 \rho}{\alpha^{3}} \frac{\xi_{1}}{1-\Gamma}\right]
$$

where we determine numerically $\Gamma \approx 0.22$.

\section{DERIVATION OF THE DIFFUSION COEFFICIENT FOR MOBILE OBSTACLES}

In the main text, we generalize our argument to mobile obstacles for which the nearest neighbor correlations need to be amended. In this case, we assume that the obstacles perform nearest neighbour random walks, with probability $\beta<1$ to jump 
at each time step. Indeed, the two independent unlocking mechanisms to escape an obstacle will lead to correlations between successive runs, in particular: (1) if the RTP tumbles and ends up with a polarity in the opposite direction from the obstacle leading to negative correlations, (2) the obstacle moves in one of the directions perpendicular with the polarity of the TP leading to positive correlations. We neglect here the correlations between successive runs if the obstacle moves in the direction of the RTP polarity; indeed, in this case, the RTP would immediately be trapped at the next step leading to correlations of the order $\ell_{p}$, negligible in front of the other correlation terms of the order of $\ell_{p}^{2}$. Thus, we can partition over both termination mechanisms and unlocking mechanisms and we obtain

$$
\left\langle\boldsymbol{a}_{i} \cdot \boldsymbol{a}_{i+1}\right\rangle=\frac{\alpha}{\alpha+\rho} \frac{\mathcal{C}_{+}-\mathcal{C}_{-}}{2 d}+\frac{\rho}{\alpha+\rho}\left[\frac{\beta^{*}}{\alpha^{*}+\beta^{*}} \mathcal{C}_{+}-\frac{\alpha^{*}}{\alpha^{*}+\beta^{*}} \frac{\mathcal{C}_{-}}{2 d-1}\right] \equiv \gamma \ell_{p}^{2}
$$

with probabilities $\alpha^{*}=\alpha(2 d-1) / 2 d$ and $\beta^{*}=\beta(2 d-1) / 2 d$. The first term in Equation S27 remains unchanged, while the second term now contains the two possible unlocking mechanisms when the RTP encounters an obstacle. If we consider the theory of persistent walks, we know that more generally the correlations decay exponentially with the distance between runs and are given by

$$
\left\langle\boldsymbol{a}_{i} \cdot \boldsymbol{a}_{i+k}\right\rangle=\ell_{p}^{2} \gamma^{|k|}
$$

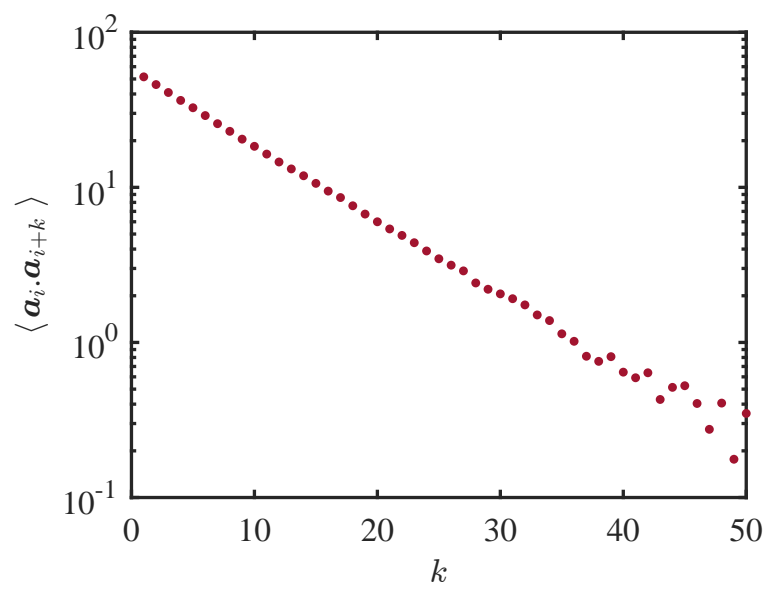

FIG. S4. Correlations between straight runs - exponential decay of correlations between straight runs averaged over 1000 realizations of simulations at obstacle density $\rho=0.1$, tumbling probability $\alpha=0.001$ and obstacle mobility $\beta=0.01$.

For instance, Figure S4 shows a clear exponential decay of these correlations in the case of a simulation with obstacle density $\rho=0.1$, tumbling probability $\alpha=0.001$ and obstacle mobility $\beta=0.01$. Thus, in the long time limit, we obtain

$$
\sum_{i \neq j}\left\langle\boldsymbol{a}_{i} \cdot \boldsymbol{a}_{j}\right\rangle=\ell_{p}^{2} \sum_{i \neq j} \gamma^{|i-j|} \underset{t \rightarrow \infty}{\sim}\langle n(t)\rangle \frac{2 \gamma}{1-\gamma} \ell_{p}^{2}
$$

Finally, in this case, the diffusion coefficient reads:

$$
\mathcal{D} \equiv \lim _{t \rightarrow \infty} \frac{\left\langle r^{2}(t)\right\rangle}{2 d t}=\frac{\bar{n}}{2 d}\left[\left\langle\boldsymbol{a}_{i}^{2}\right\rangle+\frac{2 \gamma}{1-\gamma} \ell_{p}^{2}\right]
$$

\section{NUMERICAL SIMULATIONS}

We perform Monte-Carlo simulations on a two-dimensional square lattice of step size 1. At $t=0$, the RTP is placed at the origin and we seed the lattice randomly (excluding the origin) such that the density of obstacles is uniform over the domain. For a given density $\rho$, we know that the total number of obstacles $N_{\text {obs }}$ is given by $N_{\text {obs }}=\rho L^{2}$ with $L$ the width of the lattice. To avoid finite size effect when $\alpha \rightarrow 0$, we ensure that our configurations have on average 10 obstacles per row. This imposes that $N_{\text {obs }} / L=\rho L \approx 10$. Thus, this condition dictates the total size of the lattice $L^{2}$ which we have checked to be large enough to be considered infinite for our purposes (for instance, for $\rho=0.01$ corresponds to a lattice of size $1000 \times 1000$ ). At each 
simulation time step, we start by checking for a tumble event and then we simultaneously evolve the position of the obstacles and the TP. Running times $\tau_{r}$ are defined from the moment the TP leave an obstacle, until the moment they step on the next obstacle; waiting times $\tau_{s}$ are defined from the moment the TP steps on an obstacle to the moment when it releases itself. For all data presented in the main text, statistics are obtained over at least 1000 realizations and calculated over trajectories of $10^{6}$ steps. As a consequence, the simulations were run on timescales much larger than any relevant relaxation timescale, placing our simulations in a stationary state. This process can be assumed to be ergodic, and ensemble and time averages to be equivalent.

\section{DIFFUSIVITY OF A RUN-AND-TUMBLE PARTICLE INTERACTING WITH THE OBSTACLES VIA HARD-CORE REPULSION WITH EXCLUDED VOLUME}

In the main text, we made the assumption that the size of the RTP was small compared to the size of the obstacles, leading to a trapping mechanism where the RTP can jump on a lattice site already occupied by an obstacle, but gets stuck there (see Figure S1 in Supplemental Material). To check the genericity of our results, we performed simulations in the case where the RTP cannot even enter a site occupied by an obstacle as shown on Figure S5.

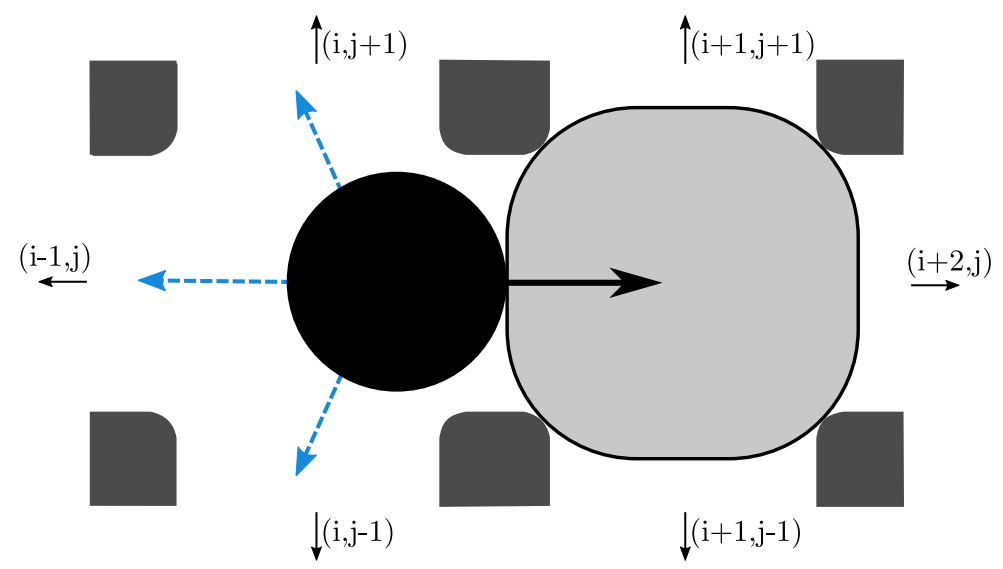

FIG. S5. Schematic description of the obstacles in the case where the RTP is not small compared to the obstacle - The RTP cannot enter the lattice site occupied by an obstacle. As for Figure S1, the obstacle is depicted in light grey, the RTP as a black disk with polarity along the black arrow and the three escape routes following a tumble of the RTP are pictured here by the dashed blue lines.

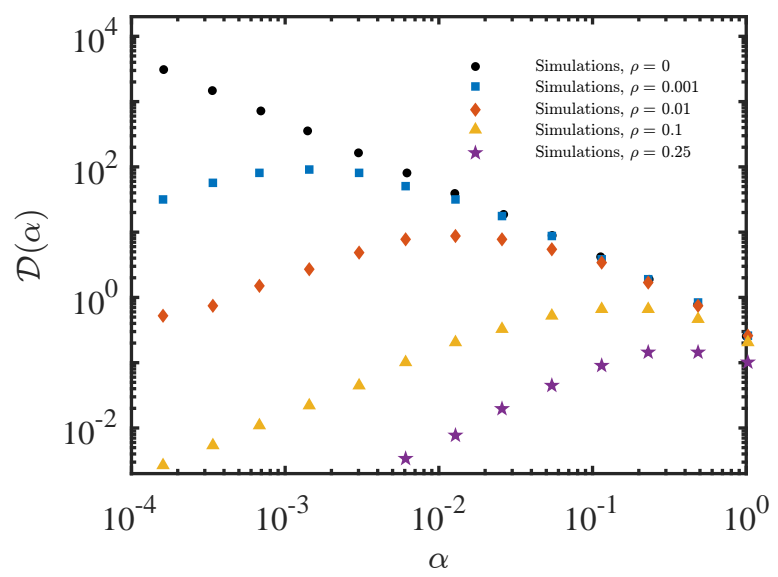

FIG. S6. Diffusivity of a run-and-tumble particle — interacting with fixed obstacles via hard-core repulsion at various obstacle densities $\rho$ as a function of tumbling probability $\alpha$. These results are qualitatively similar to results presenting in Figure 2 of the main text.

In this case, the RTP does not get trapped on the same lattice site as the trapping obstacle but rather on the neighboring lattice site. We show on Figure S6 the diffusivity of the RTP. These results are qualitatively similar to those presented in the main text; the diffusivity shows a non-monotonic behavior and displays an optimum as a function of the tumbling probability $\alpha$. The 
tumbling probability for optimal diffusivity depends on the density of obstacles $\rho$ in the same manner as shown in the main text, i.e. the optimal tumbling probability increases with the density of obstacles. It is important to note that, as shown on Figure S6, the existence of this optimal tumbling probability persists at moderate to high densities (tested numerically up to $\rho=0.25$ ). 